\title{
Relevance of multiband Jahn-Teller effects on the electron-phonon interaction in $A_{3} \mathrm{C}_{60}$
}

\author{
E. Cappelluti, ${ }^{1,2}$ P. Paci, ${ }^{2}$ C. Grimaldi, ${ }^{3}$ and L. Pietronero ${ }^{1,2}$ \\ ${ }^{1}$ INFM and "Istituto dei Sistemi Complessi"-CNR, v. dei Taurini 19, 00185 Roma, Italy \\ ${ }^{2}$ Dipartimento di Fisica, Universitá "La Sapienza”, P.le A. Moro, 2, 00185 Roma, Italy \\ ${ }^{3}$ Ecole Polytechnique Fédérale de Lausanne, LPM, Station 17, CH-1015 Lausanne, Switzerland and DPMC, Université de Genève, 24 \\ quai Ernest Ansermet, CH-1211 Genève 4, Switzerland
}

(Received 3 May 2005; published 24 August 2005)

\begin{abstract}
Assessing the effective relevance of multiband effects in fullerides is of fundamental importance in understanding the complex properties of these compounds. In this paper we investigate the role of the multiband effects on the electron-phonon (el-ph) properties of the $t_{1 u}$ bands coupled with the Jahn-Teller intramolecular $H_{g}$ vibrational modes in $\mathrm{C}_{60}$ compounds. We show that, assuming perfect degeneracy of the electronic bands, vertex diagrams arising from the breakdown of the adiabatic hypothesis, are one order of magnitude smaller than the noncrossing terms usually retained in the Migdal-Eliashberg (ME) theory. These results permit to understand the robustness of the ME theory found by numerical calculations. The effects of the nondegeneracy of the $t_{1 u}$ in realistic systems are also analyzed. Using a tight-binding model we show that the el-ph interaction is mainly dominated by intraband scattering within a single electronic band. Our results question the reliability of a degenerate band modeling and show the importance of these combined effects in the $A_{3} \mathrm{C}_{60}$ family.
\end{abstract}

DOI: $10.1103 /$ PhysRevB.72.054521

PACS number(s): 74.70.Wz, 63.20.Kr, 74.25.Kc

Although there is a large consensus about the phononmediated nature of the superconducting coupling in $A_{3} \mathrm{C}_{60}$ compounds, the precise identification of the origin of such high critical temperatures $\left(T_{c}\right.$ up to $\left.\simeq 40 \mathrm{~K}\right)$ is still an object of debate. Electron and phonon properties in these compounds are described in terms of molecular crystals, where the small hopping integral $t$ between nearest-neighbor $\mathrm{C}_{60}$ molecular orbitals sets the scale of the electronic kinetic energy, while the electron-phonon (el-ph) coupling is essentially dominated by the intramolecular vibrational modes. ${ }^{1}$ First-principle calculations suggest that el-ph coupling is mainly dominated by the $H_{g}$ modes, having energies $\omega_{H_{0}}$ $\sim 30-200 \mathrm{meV}$. A simple application of the conventional Migdal-Eliashberg (ME) theory in these materials is however questioned for different reasons. On the one hand, the small value of $t$ gives rise to strong electronic correlation effects since $t$ appears to be of the same order as the intramolecular Hubbard repulsion $U .{ }^{1}$ On the other hand, nonadiabatic effects are also expected to be relevant since the electronic energy scale $t$ is of the same order as the phonon frequency scale $\omega_{\mathrm{ph}} \cdot{ }^{2}$ In addition, the multiband nature of the electronic states $t_{1 u}$ involved in the pairing and the Jahn-Teller (JT) nature of the $H_{g}$ phonon modes make the problem even more complex but also more interesting.

A popular tool to investigate el-ph properties in systems where all the energy scales $t, U, \omega_{\mathrm{ph}}$ are of a similar order of magnitude, is the dynamical mean-field theory (DMFT), ${ }^{3}$ which does not rely on any small parameter expansion. This approach is particularly suitable in fullerides because the intramolecular el-ph interaction, just as the Hubbard repulsion, is local by construction when expressed in the basis of molecular orbitals. In order to provide a close set of equations, the hopping integrals $t_{i j}^{m m^{\prime}}$ are often chosen to be diagonal with respect to the indexes of molecular orbitals $m, m^{\prime}$, and, in the absence of crystal-field splitting, degenerate $t_{i j}^{m m^{\prime}}$ $=\delta_{m m^{\prime}} t_{i j}$. By using this approximation, the interplay between electronic correlation and el-ph coupling mediated by the JT mode has been studied. In particular, in this model multiband JT effects are claimed to be crucial to prevent the suppression of the effective superconducting pairing due to the Hubbard repulsion for small $U{ }^{4}$ accompanied by its remarkable enhancement close to the metal-insulator transition. ${ }^{5}$ Within the same approximation the validity of ME theory for a multiband JT $(t \times H)$ model has also been investigated, showing good agreement between the ME theory and DMFT results up to intermediate-large values of the el-ph coupling $\lambda \lesssim 1$, in contrast to the case of a single band system interacting with a non-JT mode $(a \times A)$, where the ME theory breaks down for $\lambda \gtrsim 0.5 .{ }^{4}$ This result is often interpreted as validity of Migdal's theorem enforcing the noncrossing approximation, which is at the basis of the ME theory. However, the numerical solution of DMFT equations makes it hard to identify the physical origin of these results, and no analytical explanation for this behavior has been so far advanced.

The aim of this paper is to provide an analytical insight into the relevance of the multiband and JT-like effects on the el-ph interaction in fullerides. In particular, we show that, assuming degenerate electronic bands, the apparent robustness of the ME theory is intrinsically related to the peculiar symmetry of the JT $H_{g}$ modes of the $\mathrm{C}_{60}$ molecules, giving rise to a drastic suppression of the el-ph scattering channels described by vertex diagrams. The physical origin of this suppression is thus deeply different from the so-called Migdal's theorem, which is ruled by the adiabatic ratio $\omega_{\mathrm{ph}} / t$. At the same time, we show that these results strongly depend on the degenerate band assumption, whereas a careful analysis of the electronic structure of the $A_{3} \mathrm{C}_{60}$ compounds points out that the effective nondegeneracy of the $t_{1 u}$ bands significantly reduces the multiband JT effects.

The simplest way to consider multiband JT effects in fullerides is to consider electrons in a tight-binding model on a 


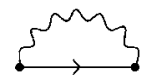

(a)

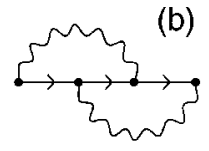

(b)

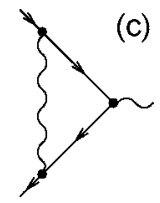

FIG. 1. Skeleton diagrams for (a) el-ph self-energy in noncrossing approximation (ME); (b) first order vertex correction to the electron-self-energy; (c) lowest order expansion of the el-ph vertex function. Solid and wavy lines are electron and phonon propagators, respectively, and filled circles the el-ph JT matrix elements. Each pictorial element is a matrix in the space of the orbital molecular index. The same diagrammatic expressions hold true even in the Nambu representation.

fcc lattice interacting with a single five-degenerate $H_{g}$ phonon mode with frequency $\omega_{0}$. Thus the Hamiltonian reads

$$
\begin{aligned}
H^{\mathrm{JT}}= & \sum_{m, m^{\prime}=1}^{3} \sum_{i j} t_{i j}^{m m^{\prime}} c_{i m}^{\dagger} c_{j m^{\prime}}+\omega_{0} \sum_{\mu=1}^{5} \sum_{i} a_{i \mu}^{\dagger} a_{i \mu} \\
& +\frac{g}{\sqrt{10}} \sum_{\mu=1}^{5} \sum_{m, m^{\prime}=1}^{3} \sum_{i} V_{m m^{\prime}}^{\mu} c_{i m^{\dagger}}^{\dagger} c_{i m^{\prime}}\left(a_{i \mu}+a_{i \mu}^{\dagger}\right),
\end{aligned}
$$

where $m, m^{\prime}$ are indexes of the molecular orbitals, $i$ is the site index, and $\mu$ labels the five-degenerate vibrational modes. $c\left(c^{\dagger}\right)$ and $a\left(a^{\dagger}\right)$ are the usual creation (annihilation) operators for electron and phonon with the quantum number specified in the Hamiltonian, and the matrices $V_{m m^{\prime}}^{\mu}$ are reported in the literature. ${ }^{6}$ The prefactor $1 / \sqrt{10}$ in the el-ph scattering term of Eq. (1) has been introduced for commodity in order to have a simple normalization of the effective coupling, namely

$$
\frac{g^{2}}{10} \sum_{\mu} \hat{V}^{\mu} \hat{V}^{\mu}=g^{2} \hat{I},
$$

but it does not play any role in the following discussion.

Let us consider for the moment the case of diagonal and degenerate hopping integrals $t_{i j}^{m m^{\prime}}=\delta_{m m^{\prime}} t_{i j}$, as it is usually done in DMFT. In this case the molecular orbital index $m$ represents a good quantum number also for the Bloch-like electron bands, which remain degenerate, and both electron Green's function $\hat{G}$ and its self-energy $\hat{\Sigma}$ thus act as the identity matrix $\hat{I}$ in the molecular orbital index $\hat{G}=G \hat{I}, \hat{\Sigma}$ $=\Sigma \hat{I}$. The el-ph matrix interaction at the ME level [Fig. 1(a)] can be evaluated in the $m \times m^{\prime}(3 \times 3)$ multiband space, and thus it reads

$$
\hat{\lambda}_{\mathrm{ME}}=\frac{\lambda_{0}}{10}\left(\begin{array}{lll}
4 & 3 & 3 \\
3 & 4 & 3 \\
3 & 3 & 4
\end{array}\right),
$$

where $\lambda_{0}=2 g^{2} N(0) / \omega_{0}$ is the single degenerate contribution from intraband and interband scattering and $N(0)$ is the electron density of states (DOS) for a single band of the threedegenerate model. The effective el-ph coupling in the normal state self-energy and in the Cooper channels are respectively given by $\lambda_{Z}^{m}=\Sigma_{m^{\prime}} \lambda_{m m^{\prime}}=\lambda_{0}$ and by the maximum eigenvalue of matrix (3), $\lambda_{\mathrm{SC}}=\max _{\text {eig }}[\hat{\lambda}]=\lambda_{0},{ }^{7}$ just as in the simple single band analysis. As a matter of fact, it is easy to see that matrix (3) can take a block diagonal form, and it behaves as a non-JT single-band system with effective el-ph coupling $\lambda_{0}$ in the one-dimensional symmetrical subspace $\widetilde{c} \equiv 1 / \sqrt{3} \Sigma_{m} c_{m}^{\dagger}|0\rangle$. Note, however, that some residual el-ph scattering with coupling $\lambda_{0} / 10$ is still operative in the orthogonal nonsymmetric space in the molecular orbital index, although it has no contribution at the ME level if the band degeneracy is preserved. As we will see, this small component with el-ph coupling $\lambda_{0} / 10$ is the only contribution surviving in the higher-order vertex diagrams.

We discuss now the robustness of the ME theory with respect to the inclusion of highest-order diagrams, i.e., vertex corrections, which are not taken into account in the ME framework. These diagrams are usually neglected in the ME theory for single-band systems by virtue of the so-called Migdal's theorem, which states that el-ph interaction processes containing vertex diagrams, as in Fig. 1(b), scale with the adiabatic parameter $\omega_{0} / t$ and are thus negligible in conventional materials where $\omega_{0} / t \ll 1 .^{8}$ These results do not apply a priori, however, in the case of $\mathrm{C}_{60}$ compounds and of other narrow-band systems where $\omega_{0} \sim t,{ }^{9}$ so that the agreement between numerical DMFT data and ME theory remains somehow surprising. As we will show, the physical origin of such agreement stems from the particular matrix structure of the $\hat{V}$ matrices in the JT multiband case of the fullerides, which leads to an almost complete cancellation of the vertex diagrams independently of the value of the adiabatic parameter $\omega_{0} / t$.

Once more, the simplest way to understand this feature is to assume a diagonal-degenerate hopping term. In order to clarify the role of multiband JT effects on the vertex processes, let us compare at the skeleton level, for both $t \times H$ and $a \times A$ models, a self-energy contribution involving a vertex diagram [Fig. 1(b)] and a typical vertex-free diagram, which is usually taken into account in the ME theory [Fig. 1(a)]. For a $a \times A$ model we can write in a compact form

$$
\begin{gathered}
\Sigma_{\mathrm{a}}^{0}(k)=-g^{2} \sum_{p} D(k-p) G(p), \\
\Sigma_{\mathrm{b}}^{0}(k)=-g^{4} \sum_{p} D(k-p) G(p) \Lambda(p, k),
\end{gathered}
$$

where $p$ is the momentum-frequency vector $\left(\mathbf{p}, \omega_{p}\right)$ and $\Sigma_{p}$ $=\Sigma_{\mathbf{p}} \int d \omega_{k} / 2 \pi$, and where $\Lambda(p, k)$ represents the lowest-order vertex correction depicted in Fig. 1(c). In the early 1960's Migdal was able to show that

$$
\lim _{\omega_{0} / t \rightarrow 0} \Lambda(p, k) \propto \frac{\omega_{0}}{t},
$$

which allows to neglect vertex diagrams in the adiabatic limit $\omega_{0} / t \ll 1$. 
The same diagrammatic picture (Fig. 1) and similar analytical expressions hold true for the multiband JT $t \times H$ model, properly generalized in the matricial space of the molecular orbital index. For the diagonal-degenerate hopping term case the electron Green's function is once more proportional to the identity matrix $\hat{I}$, so that the only matricial structure comes from the $\hat{V}$ matrices in the el-ph scattering term. In an explicit way we can write

$$
\begin{aligned}
\hat{\Sigma}_{\mathrm{a}}^{\mathrm{JT}}(k) & =-\frac{g^{2}}{10}\left[\sum_{\mu} \hat{V}^{\mu} \hat{V}^{\mu}\right] \sum_{p} D(k-p) G(p) \\
& =-g^{2} \hat{I} \sum_{p} D(k-p) G(p), \\
\hat{\Sigma}_{\mathrm{b}}^{\mathrm{JT}}(k) & =-\frac{g^{4}}{100}\left[\sum_{\mu \nu} \hat{V}^{\mu} \hat{V}^{\nu} \hat{V}^{\mu} \hat{V}^{\nu}\right] \sum_{p} D(k-p) G(p) \Lambda(p, k) \\
& =-\frac{g^{4}}{10} \hat{I} \sum_{p} D(k-p) G(p) \Lambda(p, k),
\end{aligned}
$$

where the last relation comes from the matricial properties of the $\hat{V}$ terms, $\left[\Sigma_{\mu \nu} \hat{V}^{\mu} \hat{V}^{\nu} \hat{V}^{\mu} \hat{V}^{\nu}\right] / 100=\hat{I} / 10$. We would like to stress once more that the reduction factor 10 in the last line of Eq. (8) is not related to the normalization factor in Eqs. (1) and (2), but it stems directly from the JT structure of the $\hat{V}^{\mu}$ matrices.

The comparison between Eqs. (4) and (5) and Eqs. (7) and (8) shows that a strong reduction of the vertex diagrams, of a factor 10 , is operative in the $t \times H$ model appropriate for fulleride compounds in the case of a diagonal-degenerate hopping term, validating at a large extent the noncrossing approximation well beyond the adiabatic regime. We would like to stress that the robustness of the ME theory with respect to the inclusion of vertex diagrams does not stem from the negligibility of the vertex function $\Lambda$, which can be well sizable for $\omega_{0} \sim t$, but from the noncommutativity of the $\hat{V}$ matrices and from the assumption of the diagonal and degenerate hopping term. As matter of fact, these results hold true even if the assumption of a diagonal hopping term is relaxed as long as the three electronic bands are degenerate. To show this, we can diagonalize the hopping term in Eq. (1) by the transformation

$$
c_{a}(\mathbf{k})=\sum_{i, m} M_{\mathbf{k}}^{a m} c_{i, m} \mathrm{e}^{i \mathbf{k} \cdot \mathbf{R}_{i}},
$$

so that the el-ph scattering term becomes:

$$
g \hat{V}^{\mu} \rightarrow \hat{g}_{\mathbf{k}, \mathbf{k}+\mathbf{q}}^{\mu}=g \hat{U}_{\mathbf{k}, \mathbf{k}+\mathbf{q}}^{\mu}=\hat{M}_{\mathbf{k}}^{-1} \hat{V}^{\mu} \hat{M}_{\mathbf{k}+\mathbf{q}} .
$$

Since the electron Green's function are still diagonal and degenerate $\hat{G}(k)=\hat{I} G(k)$ in this new basis, the matricial structure of the self-energy terms is still only given by the el-ph matrices $\hat{U}$. Thus we have

$$
\begin{aligned}
\frac{1}{100} \sum_{\mu \nu} \hat{U}^{\mu} \hat{U}^{\nu} \hat{U}^{\mu} \hat{U}^{\nu}= & \frac{1}{100} \sum_{\mu \nu} \hat{M}^{-1} \hat{V}^{\mu} \hat{M} \hat{M}^{-1} \\
& \times \hat{V}^{\nu} \hat{M} \hat{M}^{-1} \hat{V}^{\mu} \hat{M} \hat{M}^{-1} \hat{V}^{\nu} \hat{M} \\
& =\frac{1}{100} \hat{M}^{-1} \hat{V}^{\mu} \hat{V}^{\nu} \hat{V}^{\mu} \hat{V}^{\nu} \hat{M}=\frac{1}{10} \hat{I} .
\end{aligned}
$$

Note that a similar analysis in the double-degenerate JT $e$ $\times E$ model would predict a complete cancellation of this family of vertex processes, as previously discussed by Takada in relation to polaronic features. ${ }^{10}$

From the above discussion, one could be tempted to conclude that an effective noncrossing ME theory is expected to be enforced in fullerides due to an almost complete cancellation of the vertex processes. However, it should be stressed that the above results are valid as long as the electronic bands can be considered degenerate. In the last part of this paper, we are going to argue that this latter assumption is not appropriate in $A_{3} \mathrm{C}_{60}$ compounds in regards to el-ph properties, and a single-band model, which is unaffected by JT effects, is a better starting point for $\omega_{0} \ll t$. In order to introduce a more realistic electronic band structure than a simple diagonal-degenerate model in the molecular orbital space, we consider a tight-binding (TB) model for the $t_{1 u}$ bands as discussed in Ref. 11, which reproduces first-principle localdensity approximation (LDA) calculations with a high degree of precision. For the sake of simplicity we consider the one-directional TB model where all the $\mathrm{C}_{60}$ molecules have a fixed orientation in the fcc cystal. Different directional ordering will be discussed in a more extended publication but they are not expected to qualitatively affect our results since they still predict a set of narrow bands with only few Fermi cuts. The electronic hopping term in Eq. (1) is then diagonalized by the $\hat{M}$ matrix introduced in (9), where each matrix element depends on the electronic momentum k. For a generic point of view, the degeneracy of the electronic states in the crystal structure is preserved only on special high-symmetry points. The electronic band structure along the highsymmetry axes of the fcc Brillouin zone and the total electron density of states (DOS) has been widely reported in the literature. ${ }^{1}$ In order to assess to which extent the realistic band structure can be approximated by a degenerate threeband model, it should be noted that (low-energy) electronic and transport properties are mainly determined by quantities defined at the Fermi level, as the Fermi surface itself, the electron DOS at the Fermi level, the Fermi velocity, etc. A realistic band structure can thus be modeled as a degenerate three-band system only if it presents qualitatively similar Fermi sheets, with similar densities of states and similar Fermi velocities. In this perspective $A_{3} \mathrm{C}_{60}$ compounds seems not to be a good candidate since it is known to have only two Fermi cuts of the electronic bands with quite different Fermi surfaces. ${ }^{12}$ The smooth topological evolution of the Fermi surfaces with the Fermi level permits to identify in an unambiguous way, for what concerns electronic and transport properties, the three electronic bands with their corresponding DOS. The electronic DOS for each band, as well as the total DOS, is plotted in Fig. 2(a), which shows that the elec- 

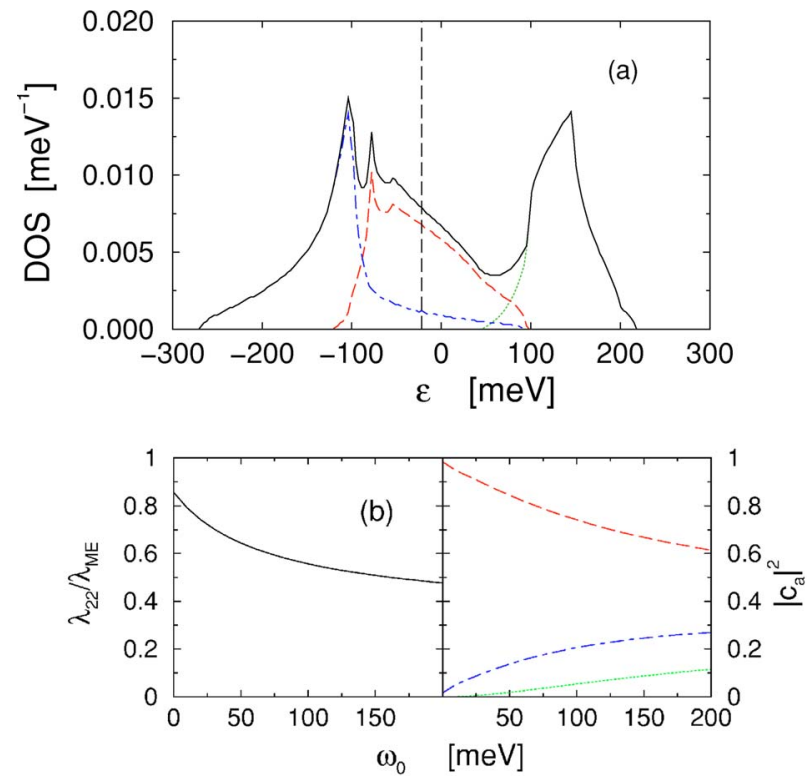

FIG. 2. (Color online) (a) Total DOS (black solid line) and partial DOS for each electronic band in the one-directional TB model for $A_{3} \mathrm{C}_{60}$. The dashed vertical line represents the Fermi level $\mu$. (b) Left panel: Ratio between the only intraband contribution $\lambda_{22}$ and the total el-ph coupling $\lambda_{\mathrm{SC}}$. Right panel: Band components $\left|c_{m}\right|^{2}$ of the eigenvector of the multiband el-ph coupling matrix $\hat{\lambda}$.

tronic band structure is largely dominated at the Fermi level, $\mu=-23 \mathrm{meV}$, by a single band that is roughly half-filled, with two additional bands that are quite far from the Fermi level. Only two bands have finite DOS at the Fermi level, in agreement with the report of only two Fermi surface. We would like to stress that the nondegeneracy of the DOS is not related to the removal of the crystal-field symmetry, but it is uniquely determined by nondegeneracy of the electronic dispersion. If we evaluated the mean value $\bar{\epsilon}=\langle\epsilon\rangle$ and the variance $\delta \epsilon=\left\langle(\epsilon-\bar{\epsilon})^{2}\right\rangle^{1 / 2}$ of each band, we obtain $\bar{\epsilon}_{1}=-120 \mathrm{meV}, \delta \epsilon_{1}=60 \mathrm{meV}, \bar{\epsilon}_{2}=-23 \mathrm{meV}, \delta \epsilon_{2}=47 \mathrm{meV}$, $\bar{\epsilon}_{3}=135 \mathrm{meV}$, and $\delta \epsilon_{3}=30 \mathrm{meV}$. These considerations suggest $A_{3} \mathrm{C}_{60}$ can be modeled in good approximation as a single-band system and that multiband effects are qualitatively irrelevant as far as the phonon energy is not sufficiently high to switch on el-ph scattering between different bands.

We sustain these intuitive arguments with a numerical calculation of the multiband el-ph matrix interaction $\hat{\lambda}_{\mathbf{k}}$ within the noncrossing approximation [Fig. 1(a)], which generalizes the ME theory to systems with generic DOS $N(\epsilon)$. For the sake of simplicity we consider unrenormalized electron and phonon propagators, with energy spectra respectively given by the TB electron dispersion and by the phonon frequency $\omega_{0}$. A k-independent el-ph interaction is obtained in the usual way by a proper average over the Fermi surface: $\lambda^{a b}$ $=\left[\Sigma_{\mathbf{k}} D\left(\epsilon_{\mathbf{k}}^{a}\right) \lambda_{\mathbf{k}}^{a b}\right] /\left[\Sigma_{\mathbf{k}} D\left(\epsilon_{\mathbf{k}}^{a}\right)\right]$, where $D(\epsilon)=(1 / \pi) \omega_{0} /\left(\omega_{0}^{2}+\epsilon^{2}\right)$, which reduces to $\Sigma_{\mathbf{k}} D\left(\epsilon_{\mathbf{k}}^{a}\right) \simeq N\left(\epsilon_{\mathrm{F}}^{a}\right)$ for $\omega_{0} \rightarrow 0$. For the sake of simplicity we neglect self-energy effects, which are expected to shrink furthermore the electron bands at the Fermi level. In order to provide a first simple estimate of multiband effects, in the left panel of Fig. 2(b) we compare the effective total el-ph coupling $\lambda_{\mathrm{SC}}$, obtained as the maximum eigenvalue of the matrix $\hat{\lambda}$, with the single intraband contribution $\lambda_{22}$ relative to the high DOS central band shown in Fig. 2(a). For $\omega_{0} \rightarrow 0, \lambda_{22} / \lambda_{\mathrm{SC}} \simeq 0.86$, pointing out that the dominant el-ph scattering comes from intraband processes with a small interband contribution due to the small DOS of the lowest electron band. As expected, the discrepancy between $\lambda_{22}$ and $\lambda_{\mathrm{SC}}$ becomes more relevant as the activation of high-energy interband processes by the phonon increases. To quantify further the role of multiband effects, we plot in the right panel of Fig. 2(b) the components of the eigenstate of the el-ph scattering matrix on the Bloch-like band index $a$. For $\omega_{0}$ $\rightarrow 0$ only the $\left|c_{2}\right|^{2}$ component is sizable, reflecting that el-ph scattering is mainly intraband. By increasing $\omega_{0}$, interband processes are gradually turned on, but, for the physical range of $\omega_{0} \leqslant 200 \mathrm{meV}$, intraband el-ph scattering within the central band are still dominant. In the opposite limit $\omega_{0} \gg W(W$ being the total electronic bandwidth) the three components become identical and the $A_{3} \mathrm{C}_{60}$ compounds are expected to behave as a degenerate three-band system.

In conclusion, in this paper we have investigated the role of the electronic structure on the multiband JT effects in the el-ph interaction in fulleride compounds. We found that a band degenerate model leads to an almost cancellation of the vertex diagrams enforcing the validity of the ME theory, as observed by DMFT data. We stress again that this result is not related to the validity of Migdal's theorem but it stems from geometrical JT reasons. On the other hand, we also show that realistic band-structure calculations suggests that the el-ph interaction in $A_{3} \mathrm{C}_{60}$ compounds is mainly dominated by intraband scattering within a single band. Note that similar conclusions are not expected to apply for electronelectron (Hubbard) interaction where relevant scattering processes are not restricted in an energy window around the Fermi level. Note also that electronic and vibrational disorder could in principle strongly mix intra- and interband el-ph scattering. The effective role of the nondegeneracy on the metal-insulator transition driven by the Hubbard repulsion and the inclusion of possible crystal-field splittings and disorder will be the future developments of the present work.

We thank M. Capone and N. Manini for fruitful discussions and the careful reading of the manuscript. This work was partially funded by the MIUR project FIRB RBAU017S8R. 
${ }^{1}$ O. Gunnarsson, Rev. Mod. Phys. 69, 575 (1997).

${ }^{2}$ E. Cappelluti, C. Grimaldi, L. Pietronero, and S. Strassler, Phys. Rev. Lett. 85, 4771 (2000).

${ }^{3}$ A. Georges et al., Rev. Mod. Phys. 68, 13 (1996).

${ }^{4}$ J. E. Han, O. Gunnarsson, and V. H. Crespi, Phys. Rev. Lett. 90, 167006 (2003).

${ }^{5}$ M. Capone et al., Science 296, 2364 (2002).

${ }^{6}$ J. E. Han, O. Gunnarsson, and V. Eyert, Phys. Rev. B 60, 6495 (1999).
${ }^{7}$ H. Suhl et al., Phys. Rev. Lett. 3, 552 (1959).

${ }^{8}$ A. B. Migdal, Sov. Phys. JETP 7, 996 (1958).

${ }^{9}$ C. Grimaldi, L. Pietronero, and S. Strassler, Phys. Rev. Lett. 75, 1158 (1995).

${ }^{10}$ Y. Takada, Phys. Rev. B 61, 8631 (2000).

${ }^{11}$ S. Satpathy, V. P. Antropov, O. K. Andersen, O. Jepsen, O. Gunnarsson, and A. I. Liechtenstein, Phys. Rev. B 46, 1773 (1992).

${ }^{12}$ S. C. Erwin and W. E. Pickett, Science 254, 842 (1991). 